of identifying disease's or patient's related characteristics to drive the optimal choice is still under investigation.

Objectives: We aimed at evaluating the effectiveness and drug survival of the biological drugs (bDMARDs) and apremilast indicated for the treatment of PSA patients with inadequate response to cDMARDs. To this purpose, PsA patients on treatment with bDMARs or Apremilast recorded into the Apulian BIOPURE registry have been retrospectively analyzed. Methods: We retrospectively assessed PsA patients, fulfilling CASPAR criteria, starting a biologic drug or apremilast from June 2016 through December 2017. At baseline and at last observation within the time frame of the study, DAPSA, PASI, ASDAS-CRP, the presence of enthesitis and dactylitis were collected. Rate of patients achieving DAPSA based remission was assessed at last observation. The persistence on the first treatment was evaluated by Kaplan-Meier survival curves. Estimated hazard ratios (HRs) of discontinuing therapy or achieving remission were assessed by multivariate stepwise backward Cox regression models, adjusting for patient demographics (gender, age) and disease characteristics (disease duration, number of prior bDMARDs, baseline DAPSA, PASI, presence of enthesitis and dactylitis, type of current drug).

Results: We recruited 450 PsA patients (268, 60\% female) with mean age $53( \pm 11)$ years, disease duration $19( \pm 13)$ months, $337(75 \%)$ naïve to bDMARDs. At baseline, 404 had peripheral joint involvement and 46 axial disease, DAPSA was $18( \pm 10)$, PASI $1.6( \pm 3)$, ASDAS-CRP 2.5 $( \pm 1), 48 / 355$ (13.5) had dactylitis and 78/231 (33\%) showed enthesitis. Table 1 shows the type of drugs. At last observation, the rate of patients who achieved the DAPSA remission was significantly higher in naïve patients $(25 \%)$ than in those bDMARDs experienced $(14 \%, p=0.02)$. The rate of drug survival was not significantly different among drugs (Figure 1). Cox-regression multivariate models showed that independent baseline factors negatively associated to drug persistence were prior bDMARD treatment $(\mathrm{HR} \quad 0.70,0.50-0.97(95 \% \mathrm{Cl}), \mathrm{p}=0.03)$ and the absence of axial disease ( $\mathrm{HR} 0.56,0.35-0.91(95 \% \mathrm{Cl}), \mathrm{p}=0.02)$. Negative predictors of DAPSA remission were prior bDMARD treatment (HR 0.40, 0.19-0.83 $(95 \% \mathrm{Cl}), \mathrm{p}=0.01)$, gender female (HR 0.51, 0.29-0.91 (95\% Cl), $\mathrm{p}=0.02)$, and the absence of dactylitis(HR $0.36,0.19-0.68(95 \% \mathrm{Cl}), \mathrm{p}=0.002)$.

Conclusion: A good rate of DAPSA remission is achievable with all the current available drugs in PSA in settings of real life. Predictors of remission and persistence on treatment were being male and naïve to prior bDMARDs. No difference among drugs was detected.

Table 1. Treatments

\begin{tabular}{lcc}
\hline & Frequency & Percentage \\
\hline Adalimumab & 80 & 17.8 \\
Certolizumab & 56 & 12.4 \\
Golimumab & 61 & 13.6 \\
Etanercept & 65 & 14.4 \\
Secukinumab & 104 & 23.1 \\
Ustekinumab & 43 & 9.6 \\
Apremilast & 41 & 9.1 \\
All & 450 & 100 \\
\hline
\end{tabular}

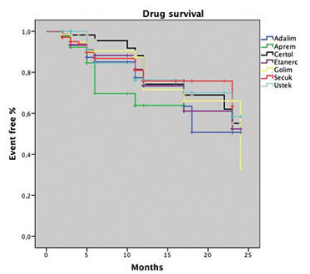

Figure 1. Kaplan-Meier curves of drug survival of PsA patients on different therapies.

Disclosure of Interests: Nicola Maruotti Speakers bureau: N Maruotti has received speaker honoraria from Pfizer outside this work, Giorgio Carlino Consultant for: G Carlino had has received consultancy fees from Pfizer, Janssen, AbbVie, MSD, BMS., Leonardo Santo Consultant for: L Santo has received consultancy fees and/or speaker honoraria from AbbVie, MSD, Novartis UCB outside this work, Speakers bureau: L Santo has received consultancy fees and/or speaker honoraria from AbbVie, MSD, Novartis UCB outside this work, Laura Quarta: None declared, Romano Bucci Consultant for: $R$ Bucci has received consultancy fees and/or speaker honoraria from Pfizer, Sanofi, MSD, BMS, Speakers bureau: R Bucci has received consultancy fees and/or speaker honoraria from
Pfizer, Sanofi, MSD, BMS, Carmelo Zuccaro Consultant for: C Zuccaro has received consultancy fees and/or speaker honoraria from MSD, AbbVie, Novartis, Pfizer, Janssen outside this work, Speakers bureau: C Zuc caro has received consultancy fees and/or speaker honoraria from MSD, AbbVie, Novartis, Pfizer, Janssen outside this work, Angelo Semeraro Speakers bureau: A Semeraro has received speaker honoraria from Sanofi, Roche, AbbVie, BMS, MSD, Novartis, Antonio Marsico: None declared, Daniela Mazzotta: None declared, Paola Chiara Francesca Falappone Consultant for: PC Falappone had received consultancy fees and/or speaker honoraria from Amgen, Abbott, MSD, BMS, outside this work, Speakers bureau: PC Falappone had received consultancy fees and/or speaker honoraria from Amgen, Abbott, MSD, BMS, outside this work, Florenzo lannone Consultant for: $F$ lannone has received consultancy fees and/or speaker honoraria from Pfizer, AbbVie, MSD, BMS Novartis, Lilly, UCB outside this work, Speakers bureau: F lannone has received consultancy fees and/or speaker honoraria from Pfizer, AbbVie, MSD, BMS, Novartis, Lilly, UCB outside this work

DOI: 10.1136/annrheumdis-2019-eular.5077

\section{FRI0450 \\ PROBABILITY OF ACHIEVING LOW DISEASE ACTIVITY OR REMISSION IN SUBJECTS WITH ACTIVE PSORIATIC ARTHRITIS TREATED WITH APREMILAST}

Philip J. Mease ${ }^{1}$, Frank Behrens ${ }^{2}$, Dafna D. Gladman ${ }^{3}$, Arthur Kavanaugh ${ }^{4}$ Michele Brunori ${ }^{5}$, Lichen Teng ${ }^{5}$, Benoit Guerette ${ }^{5}$, Ruben Queiro ${ }^{6}$, Alexis Ogdie ${ }^{7}$ ${ }^{1}$ Swedish Medical Center and University of Washington School of Medicine, Seattle, United States of America; ${ }^{2}$ Division of Rheumatology, Goethe University and Fraunhofer IME-TMP, Frankfurt, Germany; ${ }^{3} \mathrm{Krembil}$ Research Institute, Toronto Western Hospital, Toronto, Canada; ${ }^{4}$ University of California, San Diego, School of Medicine, La Jolla, United States of America; ${ }^{5}$ Celgene Corporation, Summit, United States of America; ${ }^{6}$ Rheum-Derm Unit, Hospital Universitario Central de Asturias, Oviedo, Spain; ${ }^{7}$ Perelman School of Medicine, University of Pennsylvania, Philadelphia, United States of America

Background: Therapeutic targets for long-term control of psoriatic arthritis (PsA) include the achievement of remission (REM) or low disease activity (LDA), as measured by the Clinical Disease Activity Index for Psoriatic Arthritis (CDAPSA). ${ }^{1}$

Objectives: This post hoc analysis was conducted to (1) assess the predictive values of baseline clinical disease status on achieving long-term Clinical Disease Activity for Psoriatic Arthritis (cDAPSA) targets at Week 52 and (2) examine the association between early response to APR at Week 16 and the achievement of cDAPSA targets at Week 52.

Methods: Pooled analyses of 3 phase III trials (PALACE 1-3) were performed among subjects assigned to receive APR $30 \mathrm{mg}$ twice daily at baseline. Data were analyzed using multiple imputation to account for subjects who discontinued or had missing values, using all available cDAPSA scores. The probabilities of shifting across different cDAPSA categories from baseline or Week 16 responses to Week 52 were calculated within these subjects. Binary logistic regression was also performed to confirm the results. We also analyzed mean CDAPSA from baseline to Week 52 by cDAPSA category at Week 52 among subjects with moderate or high disease activity (HDA) at baseline.

Results: A total of 494 subjects who received APR were included in the analyses; at baseline, $74.3 \%$ were in HDA, $24.5 \%$ in moderate disease activity, and $1.2 \%$ in LDA. Most subjects had $\geq 1$ of the following conditions at baseline: affected body surface area $\geq 3 \%$, dactylitis $>0$ based on the dactylitis severity score, enthesitis $>0$ based on the Maastricht Ankylosing Spondylitis Enthesitis Score or pre-existing axial PsA (physician determined) (HDA: 93.5\%; moderate disease activity: 82.6\%; LDA: 100\%). The estimated probabilities of achieving either CDAPSA LDA or REM at Week 52 were $46.9 \%$ in subjects with baseline moderate disease activity (cDAPSA $>13$ to $\leq 27$ ) and $71.1 \%$ in subjects with baseline LDA (cDAPSA $>4$ to $\leq 13$ ) (Figure). In subjects with baseline HDA (cDAPSA >27), the probability of achieving LDA or REM by Week 52 was $24.9 \%$. Subjects with moderate disease activity at baseline and improved to LDA or REM by Week 16 had high probabilities $(58.9 \%$ and $88.5 \%)$ of remaining in target at Week 52. Among the subjects with moderate disease activity at baseline, a mean CDAPSA improvement of $\geq 30 \%$ by Week 16 was associated with achievement of LDA or REM at Week 52 .

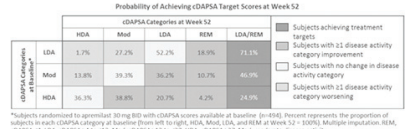


